\title{
Neue Entwicklungen in der Therapie des Ulcus cruris
}

\author{
New Developments in the Treatment of Leg Ulcers
}

Autor

Institut

\section{Moll}

Universitätsklinikum Hamburg-Eppendorf, Klinik und Poliklinik für Dermatologie und Venerologie

\section{Bibliografie}

DOI $10.1055 / \mathrm{s}-2008-1077367$

Akt Dermatol 2008; 34:

226-230 @ Georg Thieme

Verlag KG Stuttgart · New York ISSN 0340-2541

Korrespondenzadresse

\section{Prof. Dr. med. Ingrid Moll}

Universitätsklinikum Hamburg-

Eppendorf

Klinik und Poliklinik für

Dermatologie und Venerologie

Martinistr. 52

20246 Hamburg

moll@uke.uni-hamburg.de

\section{Zusammenfassung \\ $\nabla$}

Chronische Wunden betreffen 1-3\% der Bevölkerung und stellen somit ein medizinisches und soziales Problem dar. Die überwiegende Zahl sind Ulcera crurum auf Basis der chronisch venösen Insuffizienz. Neue Entwicklungen zur Therapie gibt es für alle drei Phasen der Wundheilung, Entzündungs-/Reinigungsphase, Proliferations-/ Granulationsphase und Reepithelialisierungs-/ Reparationsphase. Alle basieren auf der feuchten Wundbehandlung. Neue Verfahren zum Wunddébridement sind der niederfrequente Leistungsultraschall und die Biochirurgie, beide tragen neben Reinigung und Entfernung von Debritus zur Herstellung eines physiologischen Wundheilungsmilieus bei und induzieren dadurch die Granulation und Epithelialisierung. Hingegen erwiesen sich die modernen Wundauflagen in diesen Phasen nicht wesentlich überlegen. Neu sind zur Induktion von Granulation und Epithelialisie-

\section{Einleitung \\ $\nabla$}

Chronische Wunden stellen ein enormes medizinisches und soziales Problem dar. Der Anteil der Patienten mit chronischen Wunden wird bereits jetzt in Deutschland und anderen westlichen Industrienationen auf 2 bis $3 \%$ der Bevölkerung geschätzt. Eine weitere Zunahme läßt die demographische Überalterung der Bevölkerung erwarten $[1,2]$. Ca. $80 \%$ davon sind Ulcera crurum auf dem Boden der chronisch venösen Insuffizienz (CVI), der Rest umfasst vornehmlich diabetische Fußulzera, Dekubiti und postoperative Wunden. Oft kommen zur CVI noch arterielle Verschlusskrankheit, Diabetes mellitus oder neurologische Krankheiten hinzu. Daher erfordern Diagnostik und Therapie eine interdisziplinäre Zusammenarbeit und den Wundheilungsphasen adaptierte lokale und operative Therapien. Diese Therapien rung die Vakuumtherapie und verschiedene Wundauflagen, die wiederum nicht generell den konventionellen überlegen waren. In der Phase der Granulation sind Schaumverbände und niedrigadhäsive Wundauflagen, in der Epithelialisierung eher Hydrokolloide zu bevorzugen. Innovative Methoden wie die Applikation von Wachstumsfaktoren, Transplantation von Keratinozyten als Sheets- oder Einzelzellen und von Knochenmarksstammzellen erscheinen erfolgversprechend, sind jedoch methodisch noch nicht ausgereift. Das Ähnliche gilt für die Applikation von Inhibitoren proinflammatorischer Zytokine. Hier erwies sich ein TNF $\alpha$ monoklonaler Antikörper als erfolgreich in der Herstellung des physiologischen Wundmilieus und in der Abheilung von Ulzera. Es ist festzuhalten, dass die feuchte Wundheilung unumstritten bleibt und alle innovativen Entwicklungen dieses und die Herstellung eines physiologischen Wundmilieus implementieren.

verschlingen Behandlungskosten von mehreren Milliarden Euro pro Jahr, hinzu kommt die deutliche Minderung der Lebensqualität für die Betroffenen durch Schmerz und andere Beeinträchtigungen.

Im Folgenden soll auf neue Entwicklungen in der Therapie des Ulcus cruris fokussiert werden, die allesamt feuchte Wundbehandlung implementieren. Diese konnte sich erst ca. 30 Jahre nach ihrer Erstbeschreibung [3] in der Klinik durchsetzen, nachdem Methoden entwickelt worden waren, welche die feuchte Wundbehandlung gewährleisten und optimieren. 


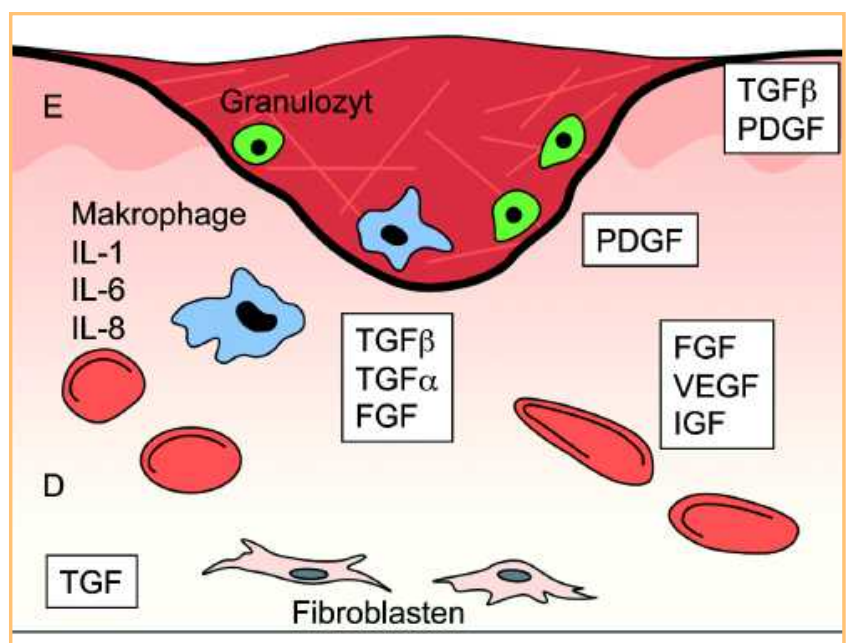

\section{E, Epidermis; D, Dermis}

Abb. 1 Akute Wundheilung. Wachstumsfaktoren aktivieren die Granulozyten, Makrophagen und Fibroblasten. TGF $\alpha / \beta$ (transforming growth factor), FGF (fibroblast growth factor), IGF (insulin like growth factor), PDGF (platelet derived growth factor), VEGF (vascular endothelial growth factor).

\section{Spontane Wundheilung}

$\nabla$

Die spontane Wundheilung ist eine komplexe Abfolge von Einzelschritten, welche zur Reepithelialisierung und Bildung von Narbengewebe führt. Sie lässt sich in 3 Phasen einteilen, die in den meisten Wunden überlappend stattfinden. In der Entzündungs-/Reinigungsphase infiltrieren neutrophile Granulozyten, Makrophagen und Lymphozyten die Wunde, deren proinflammatorische Zytokine u.a. Il-1, Il-6, Il-8 und TNF $\alpha$ zur Säuberung der Wunde führen ( $\bullet$ Abb.1). Mit der Säuberung wird über einen negativen Autoregulationsmechanismus das Sistieren der Entzündung und zugleich das Fortschreiten der Proliferation und Reparation induziert, d.h. eine spontane Wundheilung kann beginnen. In der Proliferations-/Granulationsphase beginnen Makrophagen - aus Monozyten gebildet - Wachstumsfaktoren zu produzieren, insbesondere PDGF, VEGF, EGF, FGF und TGF $\alpha$, aber auch Matrixmetalloproteasen (MMP) und ProteaseInhibitoren, z. B. Tissue Inhibitor of Metalloproteinases (TIMPs) [4-6] ( Abb. 1). Zellproliferationen laufen an, insbesondere die Granulation wird beschleunigt. Es folgt die Reepithelialisierungs-/Reparationsphase, in der die Wunde mit Epithel verschlossen und die provisorische Wundmatrix - vornehmlich aus Fibrin und Fibronektin - durch fibrotisches Bindegewebe ersetzt wird.

\section{Chronische Wunden \\ $\nabla$}

Viele aktuelle molekularbiologische Untersuchungen an chronischen Wunden argumentieren stark für Fehlregulationen der an der Wundheilung beteiligten Zellen, die ein insgesamt unphysiologisches Wundmilieu generieren mit Aktivierung von Proteasen und proinflammatorischen Zytokinen und Inaktivierung von Wachstumsfaktoren [6] ( $\bullet$ Abb.2). Die Entzündungsreaktion persistiert, möglicherweise induziert durch weiterhin vorhandenen Zelldetritus, Bakterientoxine u.a. Dadurch bleiben auch die proinflammatorischen Zytokine hochreguliert, was wiederum

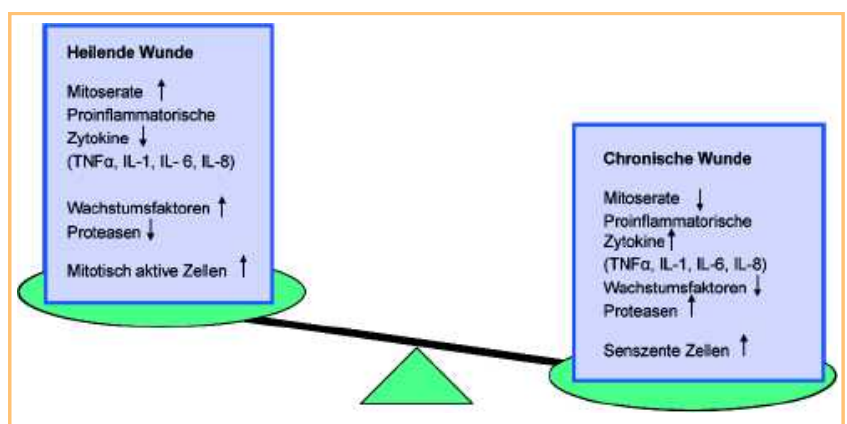

Abb. 2 Molekulare Charakteristiska von Wunden. In heilenden Wunden überwiegen die Wachstumsfaktoren, in chronischen Wunden die proinflammatorischen Zytokine und Proteasen.

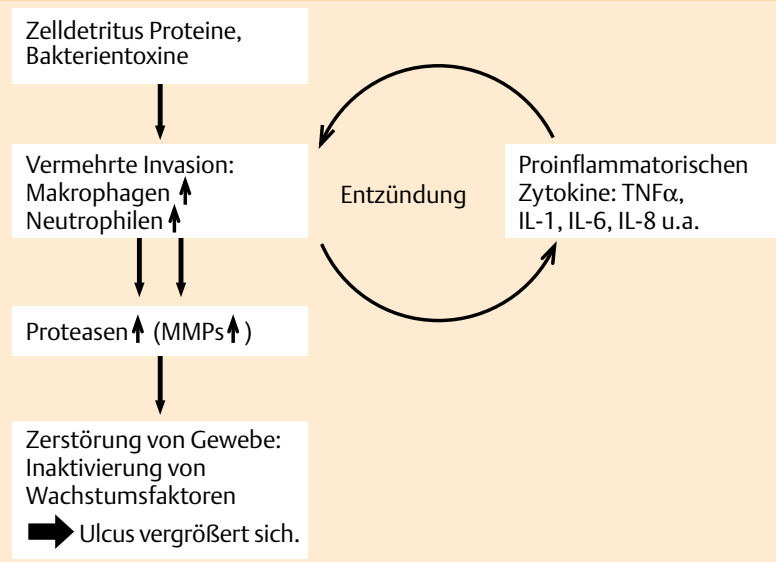

Abb. 3 Chronische Wunden. Durch Detritus, Toxine u. a. entsteht ein circulus vitiosus, der die Entzündung unterhält.

zu verstärkter Invasion von neutrophilen Granulozyten und Monozyten/Makrophagen führt, d. h. zu wiederum verstärkter Entzündung ( Abb.3). Eine sich aufschaukelnde Entzündungsreaktion hat begonnen, die auch zu verstärkter Freisetzung von Proteasen, z. B. MMPs und Elastase führt, was eine vermehrte Zerstörung von Gewebe sowie eine Inaktivierung von Wachstumsfaktoren und ihren Rezeptoren initiiert und in Folge ein Nachlassen der Proliferation. Die Ulzera werden größer. Hinzu kommen bei Infektionen bakterielle Proteasen, die ebenfalls Gewebe zerstören und Wachstumsfaktoren inhibieren ( $\bullet$ Abb. 2 und 3). Eine deutliche Vermehrung verschiedener Proteasen und proinflammatorischer Zytokine in chronischen Wunden konnte eindeutig gezeigt werden [6] ( $\bullet$ Abb. 2) und demnach ein unphysiologisches, das Zellwachstum hemmendes Wundmilieu, das sich selbst perpetuieren kann [6] ( $\bullet$ Abb.2). Eine Umstimulation des Wundmilieus ist ein Ziel aktueller Wundtherapien.

\section{Neues zum Wunddébridement $\nabla$}

\section{Niederfrequenzleistungsultraschall}

Diese neuere Methode beschleunigt das Wunddébridement durch Ultraschall $\left(25 \mathrm{kHz}, 40 \mathrm{~W} / \mathrm{cm}^{2}\right)$, der direkt auf die Wundfläche appliziert wird, versehen mit kontinuierlicher Wasserspülung, die Zelldetritus und nekrotische Reste entfernt und wegspült. Allerdings ist der Eingriff schmerzhaft und bedarf in vielen Fällen besonderer Analgesie, z. B. Lokalanästhesie. Neben 
der Abtragung von Nekrosen und Reduktion der Bakterien könnte auch eine Verstärkung der enzymatischen Autolyse, Granulation, Aktivität von Wachstumsfaktoren sowie eine höhere Effektivität von Lokaltherapeutika erreicht werden. Insgesamt resultiert daraus eine relativ rasche Reinigung des Ulcus cruris [7].

\section{Biochirurgie}

Eine andere Reinigungsmethode ist die Biochirurgie, die Verwendung von Maden der Lucilla sericata. Es stehen sehr praktische sog. Biobags zur Verfügung, in denen die Maden ausreichend Sauerstoff und Feuchtigkeit erhalten. In 48 Stunden fressen 200 Maden ca. $15 \mathrm{~g}$ nekrotisches Gewebe, dadurch sind belegte Ulzera meist in 3 Tagen sauber. Es handelt sich um eine hochselektive Nekrolyse durch Sekretion von Proteasen und Trypsin, gesundes Gewebe wird nicht angegriffen. Hinzu kommen weitere Vorteile, nämlich eine Keimreduktion, wahrscheinlich durch pH-Verschiebung und Ingestion sowie eine Stimulation des Zellwachstums durch noch unbekannte Wachstumsfaktoren im Sekret der Maden [8]. Nebenwirkungen sind die starken Schmerzen, die oft hohe Dosen an Analgetika erfordern. Maden sind besonders geeignet zur Säuberung bei Pyoderma gangraenosum und Malignomen $[1,9,10]$.

\section{Moderne Wundauflagen}

Schließlich werden zum Débridement eine zunehmende Zahl an modernen Wundauflagen propagiert. In einem systematischen Review mit Recherchen in Medline, EM BASE und Cochrane Controlled Clinical Trials Register wurden 99 relevante Studien bewertet. Generell erwiesen sich die neu entwickelten Wundauflagen nicht besser als Standardmaterialien (Kochsalzverbände oder Paraffingaze)! Beim Débridement zeigten Alginate gewisse Vorteile und sollten auch nach eigener Erfahrung bevorzugt angewendet werden [11,12].

\section{Induktion von Granulation und Epithelialisierung}

In der 2. Phase der Wundheilung, der Bildung von Granulationsgewebe mit beginnender Reepithelialisierung, spielt nach aktueller wissenschaftlicher Erkenntnis die Herstellung eines physiologischen Wundmilieus eine zentrale Rolle ( $\bullet$ Abb. 2 und 3). Ein Prinzip dazu könnte die Vakuum-Therapie (VAC-Therapie) sein ( Abb.4). Mit einem Unterdruck von 80-120 mm Hg, erzeugt durch eine Pumpe über mehrere Tage wird kontinuierlich das Wundsekret inklusive der toxischen Zerfallsprodukte wegtransportiert, das Ödem in der Wundregion reduziert, dadurch die Mikrozirkulation verbessert, sowie die Keimzahl, Schmerzen und Geruch vermindert. Verschiedene Publikationen belegen eine beschleunigte Granulation bei Ulzera [13,14]. Nebenwirkungen sind insbesondere Mazeration und Druckschäden der Umgebung, Einwachsen von Granulationsgewebe in den eingelegten Schwamm sowie starke Schmerzen und Durchblutungsminderung durch den Druck. Falls diese nicht zu beheben sind, z. B. durch Reduktion des Drucks auf $60 \mathrm{~mm}$ Hg und häufige Verbandswechsel, ist die Therapie zu beenden, um Gewebeschäden zu verhindern.

\section{Moderne Wundauflagen}

Auch in den Phasen der Granulation und Epithelialisierung erwiesen sich moderne Wundauflagen nicht eindeutig den konventionellen überlegen, Hydrokolloidverbände allerdings waren etwas überlegen, wenn die komplette Abheilung betrachtet wurde. In der Phase der Granulation sind Schaumverbände und

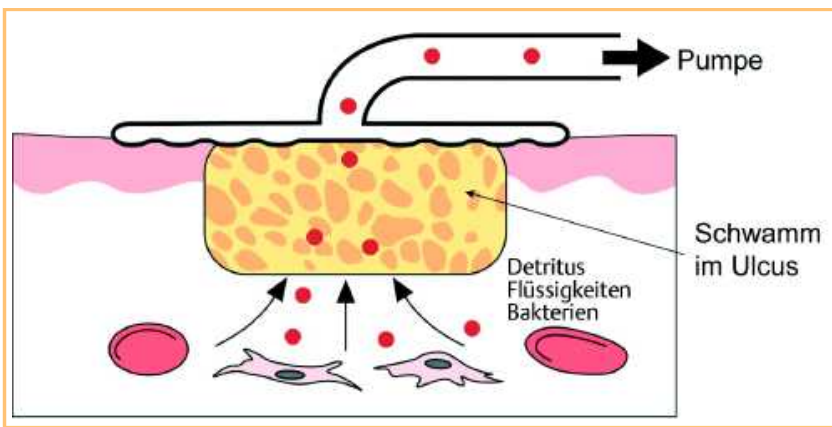

Abb. 4 Schema der VAC-Pumpe. Absaugung von Bakterien, Debritus und Flüssigkeiten u.a. kann das Wundmilieu normalisieren.

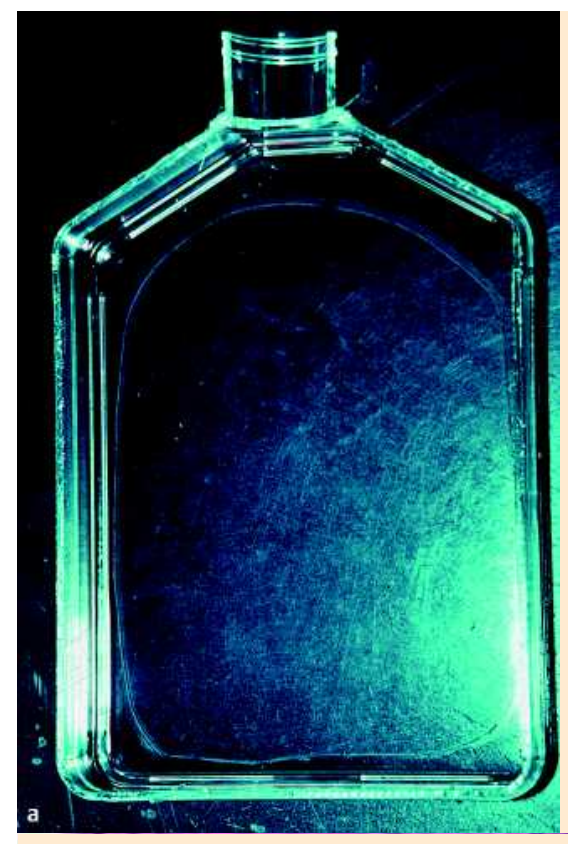

Abb. 5 Keratinozyten. a Kulturflasche mit einem KeratinozytenSheet, b Mikroskopisches Bild eines HE-gefärbten KeratinozytenSheet.

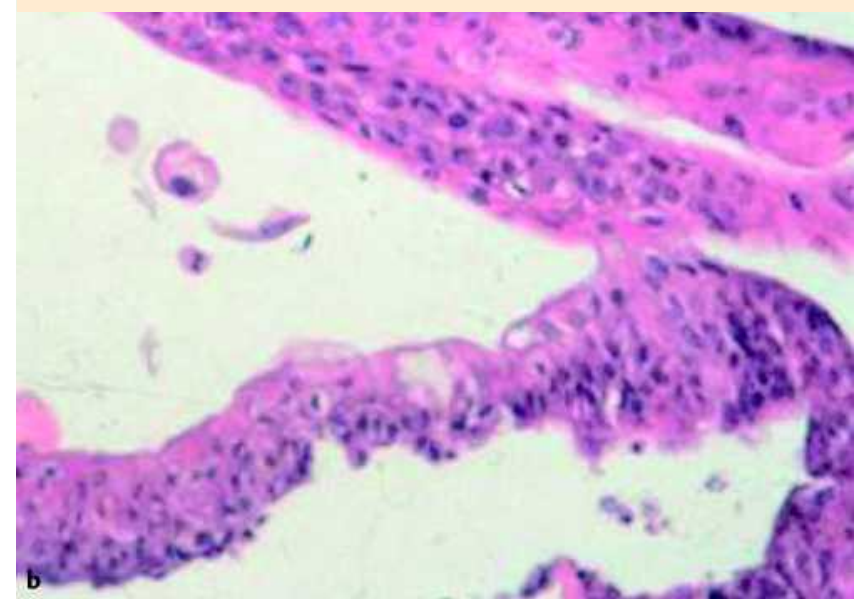

niedrig adhäsive Wundauflagen, bei betonter Reepithelialisierung eher Hydrokolloidverbände zu bevorzugen [11,12].

\section{Applikation von Wachstumsfaktoren}

Seit Mitte der 80er-Jahre existieren Erfahrungen mit der Applikation von einzelnen Wachstumsfaktoren, vornehmlich EGF, FGF, PDGF und anderen. Die überaus positiven Effekte in Zellkulturen und in Tiermodellen ließen sich bisher in keiner klinischen Studie verifizieren $[5,15]$. Lediglich in einer randomisierten doppelblin- 


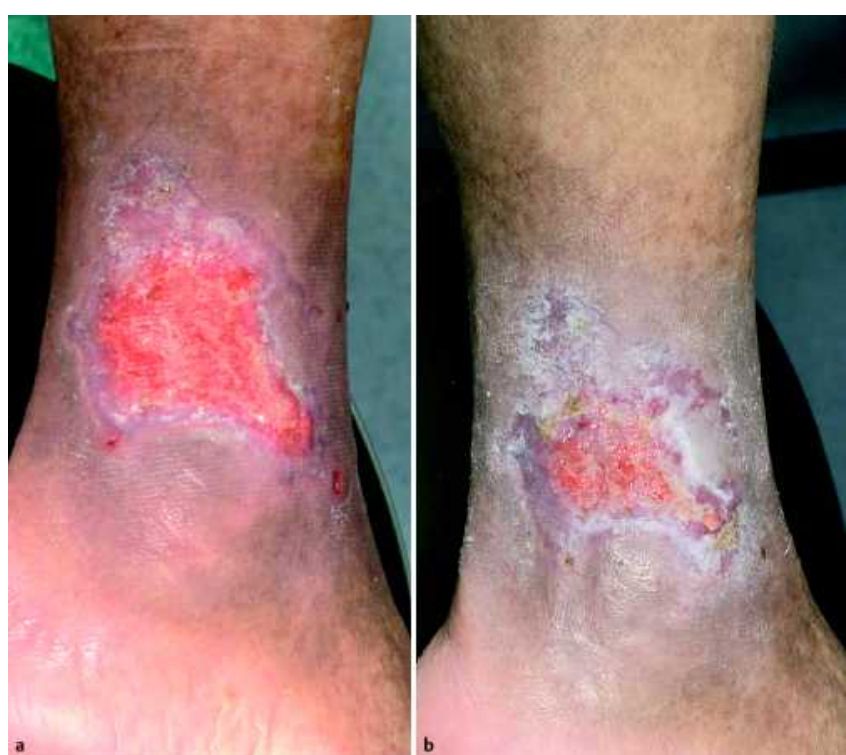

Abb. 6 Re-epithelialisierung von den Wundrändern aus (edge-effect). (a) vor, (b) 3 Wochen nach Keratinozytentransplantation auf ein Ulcus cruris venosum.

den Plazebo-kontrollierten Multicenter-Studie konnte für PDGFBB (B-Caplermin, Regranex ${ }^{\circledR}$ ) eine signifikant beschleunigte Abheilung bei diabetischen Ulzera im Vergleich zur Kontrollgruppe gezeigt werden [16]. Ob dies genauso für chronisch venös bedingte Ulzera gilt, ist derzeit noch umstritten. Auch GM-CSF könnte durch Stimulation von verschiedenen Entzündungszellen und Fibroblasten sowie Keratinozyten ( $\bullet$ Abb.5) eine wundheilungsfördernde Wirkung haben. In der Tat konnte in einer doppelblinden randomisierten Plazebokontrollierten Studie mit periläsionaler Gabe von GM-CSF in der Therapie von Ulcera crurum eine Wirkung nachgewiesen werden [17]. Jedoch sind noch weitere kontrollierte Studien nötig, um dies zu belegen. Derzeit ist festzuhalten, dass Wachstumsfaktoren in definierten Konzentrationen ein wichtiges Adjuvans eines physiologischen Wundmilieus darstellen ( $\odot$ Abb. 3). Somit könnten alle Wundauflagen, die gezielt Exsudate, Zelltrümmer, Proteasen und Ähnliches absorbieren und dadurch die Bioaktivität von Wachstumsfaktoren verlängern, einen Schritt in die richtige Richtung zu einer akuten Wundheilung darstellen. Derzeit verfügbar sind Wundauflagen mit hoher Kapillaraktivität, bestehend aus Kollagen und oxigenierter, regenerierter Cellulose (Promogran ${ }^{\circledR}$, auch verfügbar mit $1 \% \mathrm{Ag}$, Prisma ${ }^{\circledR}$ ). In einer randomisierten, prospektiven, kontrollierten Multicenter-Studie mit diabetischen Fußulzera $(n=276)$ war die Abheilungsrate in 12 Wochen der mit feuchten Gazen allerdings nicht überlegen - lediglich diabetische Ulzera, die weniger als 6 Monate bestanden hatten, zeigten eine diskret bessere Abheilungsrate [18]. Auf dem Markt befindet sich auch noch ein Azetat-Trägernetz mit Spurenelementen (polyhydrierte Ionogene, PHI 5; DerMax ${ }^{\circledR}$ ) zur Absorption von Exsudaten, Zelltrümmern und Proteasen, bei dem diese Metallionen die Zink-Ionen verdrängen und dadurch u. a. zinkabhängige Matrixmetalloproteasen inaktiviert werden. Der pH-Wert wird neutral und reaktive Sauerstoffspezies werden reduziert. Dies führte in limitierten Untersuchungen $(n=5)$ über 10 Wochen zu einer guten Abheilung von Ulzera [19].

\section{Tissue engineering}

Ein anderes Prinzip zur Induktion, Granulation und Reepithelialisierung ist die Transplantation von Zellen aus der Kultur, die ebenfalls seit Mitte der 80er-Jahre weltweit propagiert wird [20] (० Abb. 5).

Der Effekt der Transplantation von Keratinozyten scheint die Abheilung von den Wundrändern aus zu sein (edge-effect), induziert durch die Sekretion von Wachstumsfaktoren und Zytokinen FGF, EGF, PDGF, VEGF, IL-1, IL-6 u.a. ( $\bullet$ Abb. 6) D. h. Keratinozyten werden appliziert und produzieren hohe Konzentrationen an Wachstumsfaktoren. Dazu passt, dass die Applikation kleiner Sheets [21] bzw. einzelner Zellen [22] äquivalent war mit der Transplantation großflächiger Keratinozyten-Sheets [20] ( $\odot$ Abb.5). Transplantierte Zellen verändern das Verhältnis der Proteasen und Wachstumsfaktoren zugunsten der letzteren.

Die Optimierung der Zelltherapie stellt eine Herausforderung der aktuellen Wundheilungsforschung dar. Eventuell sind Knochenmarksstammzellen, die vermutlich in Keratinozyten, Fibroblasten und viele andere Zellen differenzieren können, eine weitere potenzielle Möglichkeit der Wundheilungsinduktion. Nach 1-3-maliger Injektion von Knochenmarksaspiraten und kultivierten Knochenmarkszellen konnte eine rasche Abheilung in 3 Fällen beobachtet werden [23].

Neben der Transplantation von Haut oder Knochenmarksstammzellen werden Mechanismen, die ein physiologisches Milieu initiieren, künftig eine zentrale Rolle in der Behandlung von Wundheilungsstörungen spielen. Zur Induktion eines physiologischen Wundmilieus sind derzeit viele Kandidaten in der Diskussion, verfügbar allerdings sind vor allem TNF $\alpha$-Inhibitoren. Der TNF $\alpha$-Antikörper Infliximab wurde bereits in der Therapie von Ulzera in Form eines Gels und einer Lösung eingesetzt. 14 Ulzera wurden damit behandelt und nach 8 Wochen evaluiert. 5 Ulzera waren abgeheilt, weitere 4 hatten sich um mehr als $75 \%$ reduziert, die übrigen sprachen nur mäßig oder schlecht an. Der gute Effekt des TNF $\alpha$-Antikörpers in den meisten Fällen ist hoffnungsvoll [24]. Randomisierte, doppelblinde, prospektive Studien werden folgen müssen, um die Effizienz der TNFo-Blockade durch lokale Anwendung von Antikörpern zu überprüfen. Zusammenfassend ist festzuhalten: Es gibt neue Entwicklungen in der Therapie des Ulcus cruris, beim Débridement, bei der Induktion von Granulation und Epithelialisierung. Die feuchte Wundbehandlung bleibt unumstritten und ist die Voraussetzung für die Herstellung eines physiologischen Milieus, das die akute Wundheilung charakterisiert. Alle aktuellen erfolgversprechenden Konzepte verfolgen diese beiden Prinzipien.

\section{Abstract}

\section{New Developments in the Treatment of Leg Ulcers} $\nabla$

$1-3 \%$ of the population in industrial countries suffer from chronic wounds, mostly leg ulcers due to chronic venous insufficiency. Various therapeutic innovations have been developed for three phases of wound healing, namely inflammation phase, proliferation/granulation phase, and reepithelialization phase. All are based on moist wound healing.

New methods for wound debridement are low frequency ultrasound and biosurgery with maggots. Both induce reduction of debris and induce a physiologic wound milieu, but in addition induce stimulation and reepithelialization. The VAC-therapy and in addition various bandages are a sucessful innovation. 
The latter are not superior in general to classical ones. During granulation foames and low adhesive bandages and during epithelialization hydrocolloids are preferable and recommended. Innovations such as the application of ointments containing growth factors, transplantation of keratinocytes or of bone marrow derived stem cells are hopeful but the methods have to be improved. The same may be true for application of inhibitors of pro-inflammatory cytokines, especially one TNF $\alpha$ monoclonal antibody was successful in healing leg ulcers. It has to be emphasized that moist wound healing is gold standard and all innovations include moist conditions and reconstitution of physiologic wound milieus.

\section{Literatur}

1 Karrer S. Topische Ulkustherapie [Topical therapy of ulcers]. Hautarzt 2005; 56: 1165 - 1178

2 Stücker M, Harke K, Rudolph Tet al. Zur Pathogenese des therapieresistenten Ulcus cruris. Hautarzt 2003; 54: 750 - 755

3 Winter GD. Formation of the scab and the rate of epithelization of superficial wounds in the skin of the young domestic pig. Nature 1962; 193: $293-294$

4 Eming SA. Management chronischer Wunden: Wohin geht die konservative Therapie? J Wound Healing 2007; 2: $98-102$

5 Lawrence WT, Diegelmann RF. Growth factors in wound healing. Clin Dermatol 1994; 12: 157-169

6 Lobmann R, Schultz G, Lehnert H. Proteases and the diabetic foot syndrome: mechanisms and therapeutic implications. Diabetes Care 2005; $28: 461-471$

7 Liebold $K$, Uhlemann C, Wollina U. Ulcus-Debridement mit niederfrequentem Ultraschall. ZfW 2001; 18: 20-22

8 Brandner JM, Houdek P, Quitschau V et al. An ex-vivo model to evaluate dressing \& drugs for wound healing. EWMA 2006; 6: 11-15

9 Wollina $U$, Karte $K$, Herold $C$ et al. Biosurgery in wound healing - the renaissance of maggot therapy. J Eur Acad Dermatol Venereol 2000; 14: $285-289$

10 Singer AJ, Clark RA. Cutaneous wound healing. N Engl J Med 1999; 341 : $738-746$
11 Chaby G, Senet $P$, Vaneau $M$ et al. Dressings for acute and chronic wounds: a systematic review. Arch Dermatol 2007; 143: 1297-1304

12 Vaneau M, Chaby G, Guillot B et al. Consensus panel recommendations for chronic and acute wound dressings. Arch Dermatol 2007; 143: $1291-1294$

13 Fleischmann W, Russ M, Westhauser A et al. Die Vakuumversiegelung als Trägersystem für eine gezielte lokale Medikamentenapplikation bei Wundinfektionen. Unfallchirurg 1998; 101: 649-654

14 Trent JT, Falabella A, Eaglstein WH et al. Venous ulcers: pathophysiology and treatment options. Ostomy Wound Manage 2005; 51: 38 -54; quiz 55-36

15 Meyer-Ingold $W$. Wound therapy: growth factors as agents to promote healing. Trends Biotechnol 1993; 11: 387-392

16 Wieman TJ, Smiell JM, Su Y. Efficacy and safety of a topical gel formulation of recombinant human platelet-derived growth factor-BB (becaplermin) in patients with chronic neuropathic diabetic ulcers. A phase III randomized placebo-controlled double-blind study. Diabetes Care 1998; $21: 822-827$

17 Marques da Costa R, Jesus FM, Aniceto C et al. Double-blind randomized placebo-controlled trial of the use of granulocyte-macrophage colony-stimulating factor in chronic leg ulcers. Am J Surg 1997; 173: $165-168$

18 Veves A, Sheehan P, Pham HT. A randomized, controlled trial of Promogran (a collagen/oxidized regenerated cellulose dressing) vs standard treatment in the management of diabetic foot ulcers. Arch Surg 2002; 137: $822-827$

19 Körber A, Freise J, Rietkötter J et al. Erfolgreiche Behandlung therapierefraktärer chronischer Wunden mit DerMax. ZfW 2006; 6: 310-314

20 Schönfeld M, Moll I, Maier K et al. Keratinocytes from cell culture for therapy of skin defects. Review and personal results. Hautarzt 1993; 44: $281-289$

21 Limat A, Hunziker T. Use of epidermal equivalents generated from follicular outer root sheath cells in vitro and for autologous grafting of chronic wounds. Cells Tissues Organs 2002; 172: 79-85

22 Moll I, Schönfeld M, Jung EG. Use of keratinocytes in therapy of ulcera crurum. Hautarzt 1995; 46: 548-552

23 Badiavas EV, Falanga V. Treatment of chronic wounds with bone marrow-derived cells. Arch Dermatol 2003; 139: 510-516

24 Streit M, Beleznay Z, Braathen LR. Topical application of the tumour necrosis factor-alpha antibody infliximab improves healing of chronic wounds. Int Wound J 2006; 3: 171 - 179 